\title{
A Step Towards Refining and Translating B Control Annotations to Handel-C
}

\author{
Wilson Ifill ${ }^{a, b}$ and Steve Schneider ${ }^{b}$ \\ ${ }^{a}$ AWE Aldermaston, Reading, Berks, England; Email: wil.ifill@awe.co.uk \\ ${ }^{\mathrm{b}}$ Department of Computing, University of Surrey, Guildford, Surrey, England. \\ \{W.Ifill, S.Schneider \} @surrey.ac.uk
}

\begin{abstract}
Research augmenting B machines presented at B2007 has demonstrated how fragments of control flow expressed as annotations can be added to associated machine operations, and shown to be consistent. This enables designers' understanding about local relationships between successive operations to be captured at the point the operations are written, and used later when the controller is developed. This paper introduces several new annotations and I/O into the framework to take advantage of hardware's parallelism and to facilitate refinement and translation. To support the new annotations additional CSP control operations are added to the control language that now includes: recursion, prefixing, external choice, if-then-else, and sequencing. We informally sketch out a translation to Handel-C for prototyping.
\end{abstract}

Keywords. B Metrhod, CSP, Hardware Description Language,

\section{Introduction}

Annotating B-Method specifications with control flow directives enables engineers to describe many aspects of design within a single notation. We generate proof obligations (pob's) to demonstrate that the set of executions allowable by the annotations of a B [1] [15] machine do not cause operations to diverge. The benefit of this approach is that only the semantics of the machine operations are required in checking the annotations, and these checks are similar in size and difficulty to standard B machine consistency checks. Controllers written in CSP, which describe the flow of control explicitly, can be checked against the annotations. There is no need to check the CSP [7] [13] [14] directly against the full B description. Once the annotations are shown to be correct with respect to the B machine we can evaluate controllers against the annotations without further reference to the machine. Machines can be refined and implemented in the normal way while remaining consistent with the controller. In previous work [9] we presented the NEXT and FROM annotations, which permitted simple annotated B specifications and controllers to be written. Before that [10] we presented a route to VHDL [5], a hardware description language, from B. In this paper we present three more annoations: NEXT_SEQ, NEXT_PAR and NEXT_COND and add input andoutput to the operations. We also begin to present an informal refinement theory for annotations and a route to implementation via HandelC. The refinement theory outline in this paper allows the annotations to be independently refined and remain consistent with the Machine.

Previous work obtaining hardware implementations from B approached the problem by using B as a Hardware Description Language (HDL) that translates to VHDL [8] [?]. Our approach 
achieves the goal of obtaining hardware via Handel-C as an intermediate stepping stone, which means that the $B$ that is translated does not require the same degree of HDL structural conformance as does the B for VHDL translation. Approaches that translate HDLs to B for analysis [4] do not support the development process directly. Event B [3] has been used to support the development of hardware circuits [2] that includes refinement but not the code generation process. Not only are we working towards code generation, but we wish to work with specifications that model both state and control equally strongly. CSP $\|$ B [18] [17] has the capability to model state and event behaviour, but the CSP controller must be instantiated with B components to verify the combination. We break the verification of controllers down into manageable stages, and offer an approach to refinement and translation. Integrations of CSP-Z by Moto and Sampaio [11] and CSP-OZ Fischer [6] require a CSP semantics to be given to $\mathrm{Z}$ in order for integration to be analyseableas awhole. Our approach differs to other formal language integrations in two ways. Firstly, The control flow behaviour is capture during the development of the state operation in the form of annotation. The annotations are control specifications. Only later is a complete controller developed that satisfies the annotations. In this way the developer of the state operations in B can constrain controller behaviour, but full controller development can be posponed. and possible performed by a different engineer. Secondly, there is no notion of executing the models together and analysing this integration for deadlocks. In this approach the different formal notations provide different views of the system, and both views are required to obtain a executable model.

This paper describes extensions to the work presented in B2007 [9]. This papers contribution is the introduction of additional next annotations, incorporation of I/O into the annotations, and an informal treatment of refinement and translation. In Section 1, the general framework is introduced. In Section 2 a B machine is introduced along with the NEXT annotation. The proof obligations associated with the annotations and control language are given in Section 3. The consistency of the annotations are given in Section 4. A refinement and translation outline is given in Section 5. An example illustration of some refinements and translations are given in section 6. A discussion on the benefits and future work is had in Section 7.

We restrict our attention in this paper to correct B machines: those for which all proof obligations have already been discharged. We use $I$ to refer to the invariant of the machine, $T$ to refer to the machine's initialisation, $P_{i}$ to refer to the precondition of operation $O p_{i}$, and $B_{i}$ to refer to the body of operation $O p_{i}$.

Controllers will be written in a simple subset of the CSP process algebraic language $[7,14]$. The language will be explained as it is introduced. Controllers are considered as processes performing events, which correspond to operations in the controlled B machine. Thus operation names will appear in the controller descriptions as well as the B machine definitions. The Handel-C translations are shallow and in a few cases performed in accordance with existing translation work [12] [16].

\section{The General Framework}

The approach proposed in this paper introduces annotations on B operations as a mechanism for bridging the gap between B machines and CSP controllers, while maintaining the separation of concerns. The approach consists of the following components:

- Machine definition: the controlled component must first be defined.

- Annotations: the initialisation and the operations in the machine definition are annotated with fragments of control flow. 
- Annotation proof obligations: verification conditions that establish consistency of the annotations with the controlled machine. This means that the fragments of control flow captured by the annotations really are appropriate for the machine.

- Controller: this is a process that describes the overall flow of control for the B machine.

- Consistency checking: establishing that the controller is consistent with the annotations by showing that that every part of the control flow is supported by some annotation.

- Refine/Translate: refinement may be needed before a translations can be achieved. The translation is the final step and requires additional annotation directives to set type sizes and $\mathrm{I} / \mathrm{O}$ ports.

Checking a CSP controller against a machine is thus reduced to checking it against the annotations and verifying that the annotations are appropriate for the machine. The relationship between the different parts of the approach are given in Figure 1.

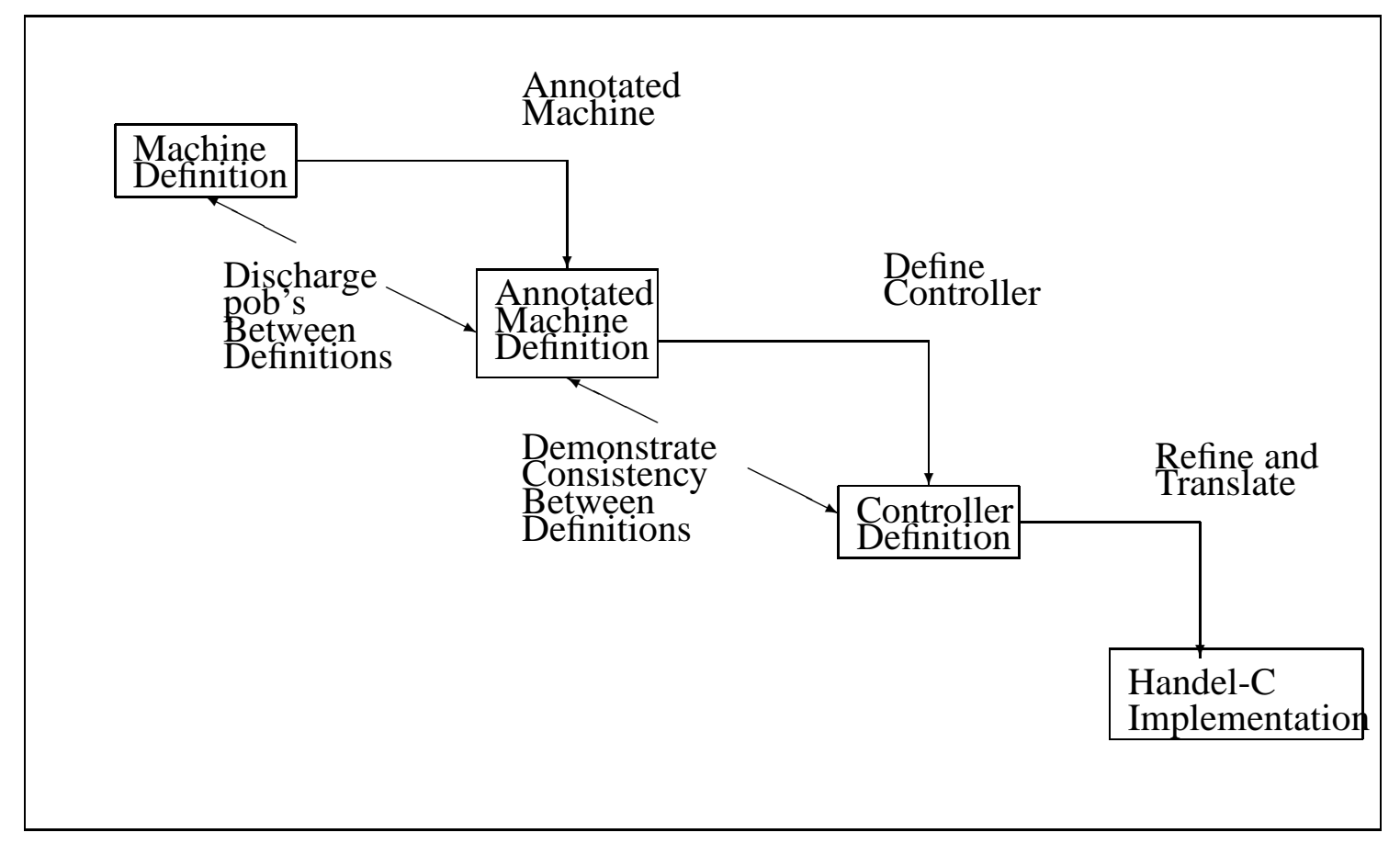

Figure 1. The Process Flow in the Approach.

The framework presented here is quite general, in that it may be applied to both Event-B and classical B. Additional annotations maybe added along with supporting control opedrations as required. Provided that a consistency argument can be developed. The first step to be taken is therefore to fix on the control language and the associated annotations to be incorporated into the $\mathrm{B}$ machine descriptions.

\section{The Approach}

We will demonstrate the approach with a simple model to illustrate aspects of the approach. The annotation we consider is the NEXT annotation. An extremely simple controller language consisting only of prefixing, choice, parallel, if-then-else, and recursion is used to develop the example. 
B-Method [1] has evolved two major approaches: traditional B and Event-B. Annotations can be used in either traditional B machines, or Event-B systems. Traditional B approaches specification in a state-oriented fashion. It focuses on the services that a system might provide, whereas Event-B focuses on the events that occur within the system. B Machines are used in the examples. The generic traditional B MACHINE $S$, given below, has variables, invariant, initialisation, and a set of operations $O P 1$ through to $O P n$ that have inputs and outputs. $v$ describes a set of inputs and $y$ describes a set of outputs to and from a operation, rerspectively.

\author{
MACHINE S \\ VARIABLES $v$ \\ INVARIANT $v$ \\ INITIALISATION $v: \in u$ \\ OPERATIONS \\ $y \longleftarrow O P 1\left(z_{1}\right) \widehat{=} P_{1} \mid B_{1}$ \\ $y_{2} \longleftarrow O P 2\left(z_{2}\right) \hat{=} G_{2} \Longrightarrow B_{2}$; \\ $y_{n} \longleftarrow O P n\left(z_{n}\right) \widehat{=} P_{n} \mid B_{n}$
}

END

The operations are defined in Guarded Substitution Language (GSL). It is asserted that the machine is consistent when each operation can be shown to establish the machine invariant, $I$, and the machine cannot deadlock. Every operation must be either guarded, $G$, or have a precondition, $P$, but all must have a next annotation (not shown). In Event-B, unlike classical $\mathrm{B}$, new operations can be added during refinement. In the example we anticipate the need for operations in the later stages of refinement by introducing the signature of the operation with a body defined by the skip operation. We do not in this paper adapt the pobs for Event-B refinement. The refinement process may involve adding detail to the specification in a consistent way to realise an implementation, which is a key notion in B. Refinement involves removing non-determinism and adopting concrete types. We add to the concept of B refinement with the annotations, by adding the notion of annotation control flow refinement.

\title{
3. The Annotation with $\mathbf{I} / O$
}

We annotate operations of a B machines with a NEXT annotation that supports operations with I/O. If the conjunction of pob's for all the annotations are discharged then we say that the annotations are consistent with the machine. A consistent controller that evolves in accordance with the next annotations steps will not diverge or deadlock. A NEXT annotation on the current operation $O P_{i}$ (where $O P_{i}$ represents $y_{i} \longleftarrow O p_{i}\left(z_{i}\right)$ and $y_{i}$ is the output vector, $y_{1} \ldots y_{n}$, and $z_{i}$ is the input parameter vector, $\left.z_{1} \ldots z_{m}\right)$ introduces another operation $O P_{j}$, or set of operations $O P_{j_{1}}, \ldots, O P_{j_{n}}$, which will be enabled after $O P_{i}$ is executed (where an operation in the annotation $O P_{j}$ represents $O p_{j}\left(e_{j}\right)$ and $e_{j}$ is the input expression vector, $\left.e_{1} \ldots e_{m}\right)$. In the NEXT annotation $e_{j}$ is a list of expressions which serves as inputs on which $O P_{j}$ can be called next. In this paper we will restrict the expressions to variables $v^{\prime} s$ defined in the B 
machines which will supply inputs in the hardware implementation. The value of this variable is not considered when calculating the pob's. Only the type of the variable used in the annotation is checked.

\subsection{The Basic NEXT Annotation}

$$
O P_{i} \widehat{=} \text { PRE } \quad P_{i} \quad \text { THEN } \quad B_{i} \text { END } \quad / *\left\{O P_{j_{1}}, \ldots, O P_{j_{n}}\right\} \text { NEXT } * / ;
$$

Definition 3.1 (Proof Obligations of the Basic NEXT on INITIALISATION) Given the following B initialisation:

\section{INITIALISATION $\mathrm{T} / *\left\{O p_{j} ? v_{j}\right\} N E X T ; * /$}

The following pob's arises: $\quad[T]\left(\left(v_{j} \in T_{j}\right) \Rightarrow P_{j}\right)$

The NEXT annotation following the initialisation indicates the first enabled operation. There can be more than one operation in the annotation. The example illustrates only one next operation. The variables used as input parameters in the annotation $\left(? v_{j_{1}} \ldots ? v_{j_{m}}\right)$ must be of the type required in the operation definition.

Definition 3.2 (Proof Obligations of the Basic NEXT on Operations) Given the following $B$ operation:

$$
\begin{aligned}
& y_{i} \longleftarrow O p_{i}\left(z_{i}\right) \stackrel{\text { PRE }}{=} P_{i} \quad \text { THEN } \quad B_{i} \quad \text { END } \\
& / *\left\{O p_{j}\left(v_{j_{1}}\right), \ldots, O p_{j_{n}}\left(v_{j_{n}}\right)\right\} N E X T * / ;
\end{aligned}
$$

The related pob's follow:

$$
\begin{aligned}
& \left(P_{i} \wedge I \Rightarrow\left[B_{i}\right]\left(\left(v_{j_{1}} \in T_{j_{1}}\right) \Rightarrow P_{j_{1}}\right)\right) \wedge \\
& \ldots \\
& \left(P_{i} \wedge I \Rightarrow\left[B_{i}\right]\left(\left(v_{j_{n}} \in T_{j_{n}}\right) \Rightarrow P_{j_{n}}\right)\right)
\end{aligned}
$$

where the elements of $v_{i}$ and $v_{j}$ are free in $B_{i}, P_{i}$, and I

\subsection{The NEXT_PAR Annotation}

I/O operations can be annotated to indicate parallel execution NEXT_PAR . Two or more sets are introduced (only two illustrated below). Any operation of a respective set can run in parallel with any other operation from any of the other sets.

Definition 3.3 (Proof Obligations of NEXT_PAR) Given the following B operation:

$$
\begin{aligned}
y_{i} \longleftarrow O p_{i}\left(z_{i}\right) \hat{=} \text { PRE } & P_{i} \quad \text { THEN } \quad B_{i} \quad \text { END } \\
/ * & \left\{O p_{j_{1}}\left(v_{j_{1}}\right), \ldots, O p_{j_{n}}\left(v_{j_{n}}\right)\right\} \\
& \left\{O p_{p_{1}}\left(v_{p_{1}}\right), \ldots, O p_{p_{n}}\left(v_{p_{n}}\right)\right\} N E X T \_P A R * / ;
\end{aligned}
$$


The related pob's follow:

$$
\begin{aligned}
& \left(P_{i} \wedge I \Rightarrow\left[B_{i}\right]\left(\left(v_{j_{1}} \in T_{j_{1}}\right) \Rightarrow P_{j_{1}}\right)\right) \wedge \\
& \ldots \\
& \left(P_{i} \wedge I \Rightarrow\left[B_{i}\right]\left(\left(v_{j_{n}} \in T_{j_{n}}\right) \Rightarrow P_{j_{n}}\right)\right) \wedge \\
& \left(P_{i} \wedge I \Rightarrow\left[B_{i}\right]\left(\left(v_{p_{1}} \in T_{p_{1}}\right) \Rightarrow P_{p_{1}}\right)\right) \wedge \\
& \cdots \\
& \left(P_{i} \wedge I \Rightarrow\left[B_{i}\right]\left(\left(v_{p_{n}} \in T_{p_{n}}\right) \Rightarrow P_{p_{n}}\right)\right) \wedge
\end{aligned}
$$

$\operatorname{variable\_ used}\left(\left\{O P_{j_{1}}, \ldots, O P_{j_{n}}\right\}\right) \cap \operatorname{variable\_ used}\left(\left\{O p_{p_{1}}, \ldots, O P_{p_{n}}\right\}\right)=\{\}$

The parallel annotation offers the option to execute two or more operations in parallel after the current operation, provided they do not set or read any variables in common. The proof obligation ensures that all the operations in the annotations are enabled after the current operation. Only one from each set will be executed in parallel.

\subsection{The NEXT_SEQ Annotation}

Operations can be annotated to indicate a requirement for a particular sequential execution: NEXT_SEQ.

Definition 3.4 (Proof Obligations of NEXT_SEQ) Given the following B operation:

$$
\begin{aligned}
y_{i} \longleftarrow O p_{i}\left(z_{i}\right) \hat{=} \text { PRE } & P_{i} \quad \text { THEN } \quad B_{i} \quad \text { END } \\
/ * & \left\{O p_{j_{1}}\left(v_{j_{1}}\right), \ldots, O p_{j_{n}}\left(v_{j_{n}}\right)\right\} \\
& \left\{O p_{p_{1}}\left(v_{p_{1}}\right), \ldots, O p_{p_{n}}\left(v_{p_{n}}\right)\right\} N E X T \_S E Q * / ;
\end{aligned}
$$

The related pob's follow:

$$
\begin{aligned}
& \left(P_{i} \wedge I \Rightarrow\left[B_{i}\right]\left(\left(v_{j_{1}} \in T_{j_{1}}\right) \Rightarrow P_{j_{1}}\right)\right) \wedge \\
& \ldots \\
& \left(P_{i} \wedge I \Rightarrow\left[B_{i}\right]\left(\left(v_{j_{n}} \in T_{j_{n}}\right) \Rightarrow P_{j_{n}}\right)\right) \wedge \\
& \left(P_{j_{1}} \wedge I \Rightarrow\left[B_{j_{1}}\right]\left(\left(v_{p_{1}} \in T_{p_{1}}\right) \Rightarrow P_{p_{1}}\right)\right) \wedge \\
& \ldots \\
& \left(P_{j_{1}} \wedge I \Rightarrow\left[B_{j_{1}}\right]\left(\left(v_{p_{n}} \in T_{p_{n}}\right) \Rightarrow P_{p_{n}}\right)\right) \wedge
\end{aligned}
$$




$$
\begin{aligned}
& \left(P_{j_{n}} \wedge I \Rightarrow\left[B_{j_{n}}\right]\left(\left(v_{p_{1}} \in T_{p_{1}}\right) \Rightarrow P_{p_{1}}\right)\right) \wedge \\
& \ldots \\
& \left(P_{j_{n}} \wedge I \Rightarrow\left[B_{j_{n}}\right]\left(\left(v_{p_{n}} \in T_{p_{n}}\right) \Rightarrow P_{p_{n}}\right)\right)
\end{aligned}
$$

where the elements of $z_{i}$ and $v_{j}$ and $v_{p}$ are free in $B_{i}, P_{i}$, and $\mathrm{I}$

The NEXT_SEQ annotation is conceptually different from the NEXT annotation, because it captures specific paths of executions that must exist in a controller. The current operation $O p_{i}$ must enable each operation in $\left\{O p_{j_{1}}\left(v_{j_{1}}\right), \ldots, O p_{j_{n}}\left(v_{j_{n}}\right)\right\}$, and each operation in that set must enable each operation in the set $\left\{O p_{p_{1}}\left(v_{p_{1}}\right), \ldots, O p_{p_{n}}\left(v_{p_{n}}\right)\right\}$. Practically, this annotation should be used to depict particular paths: one operation per set.

\subsection{The NEXT_COND Annotation}

To enable the current operation to conditionally select one set of operations next as opposed to some other set the NEXT_COND annotation is used. The condition NEXT_COND annotation is an extension to the NEXT annotation that supports conditional next path selection.

In definition 3.5 if the output of the current operation is true then all the operations $O P_{j_{1}}$ through to $O P_{j_{n}}$ are guaranteed to be available to execute. If however the current operation returns false then the operations $O P_{p_{1}}$ through to $O P_{p_{n}}$ are guaranteed to be available to execute. The proof of this claim can be verified by discharging the following proof obligation given in definition 3.5:

Definition 3.5 (Proof Obligation of NEXT_COND) Given the following B operation:

$$
\begin{aligned}
y_{i} \longleftarrow O p_{i}\left(z_{i}\right) \cong \text { PRE } & P_{i} \quad \text { THEN } \quad B_{i} \quad \text { END } \\
/ * & \left\{O p_{j_{1}}\left(v_{j_{1}}\right), \ldots, O p_{j_{n}}\left(v_{j_{n}}\right)\right\} \\
& \left\{O p_{p_{1}}\left(v_{p_{1}}\right), \ldots, O p_{p_{n}}\left(v_{p_{n}}\right)\right\} \text { NEXT_COND */; }
\end{aligned}
$$

The related pob's follow:

$$
\begin{aligned}
\left(I \wedge P_{i} \Rightarrow\right. & {\left.\left[B_{i}\right]\left(\left(y_{i}=\text { TRUE } \wedge v_{j_{1}} \in T_{j_{1}}\right) \Rightarrow P_{j_{1}}\right)\right) } \\
& \ldots \\
\wedge\left(I \wedge P_{i} \Rightarrow\right. & {\left.\left[B_{i}\right]\left(\left(y_{i}=T R U E \wedge v_{j_{n}} \in T_{j_{n}}\right) \Rightarrow P_{j_{n}}\right)\right) } \\
\wedge\left(I \wedge P_{i} \Rightarrow\right. & {\left.\left.\left[B_{i}\right]\left(\left(y_{i}=\text { FALSE }\right\} \wedge v_{p_{1}} \in T_{p_{1}}\right) \Rightarrow P_{p_{1}}\right)\right) } \\
& \ldots \\
\wedge & \left(I \wedge P_{1 i} \Rightarrow\left[B_{i}\right]\left(\left(y_{i}=\text { FALSE } \wedge v_{p_{n}} \in T_{p_{n}}\right) \Rightarrow P_{p_{n}}\right)\right)
\end{aligned}
$$

The lists of the NEXT_COND annotation do not have to be the same size. The operation that carries this annotation must have a single boolean output. 
The next annotation represents a control fragment specification of the whole system. The CSP controller represents a refined view of the annotated B system. The annotated B machine hasn't the fidelity to clearly portray the necessary control detail that the CSP can: the annotations are not clearly laid out as a set of recursive definitions. On translation both the B and the CSP are used to build the implementation, hence the need to develop a controller.

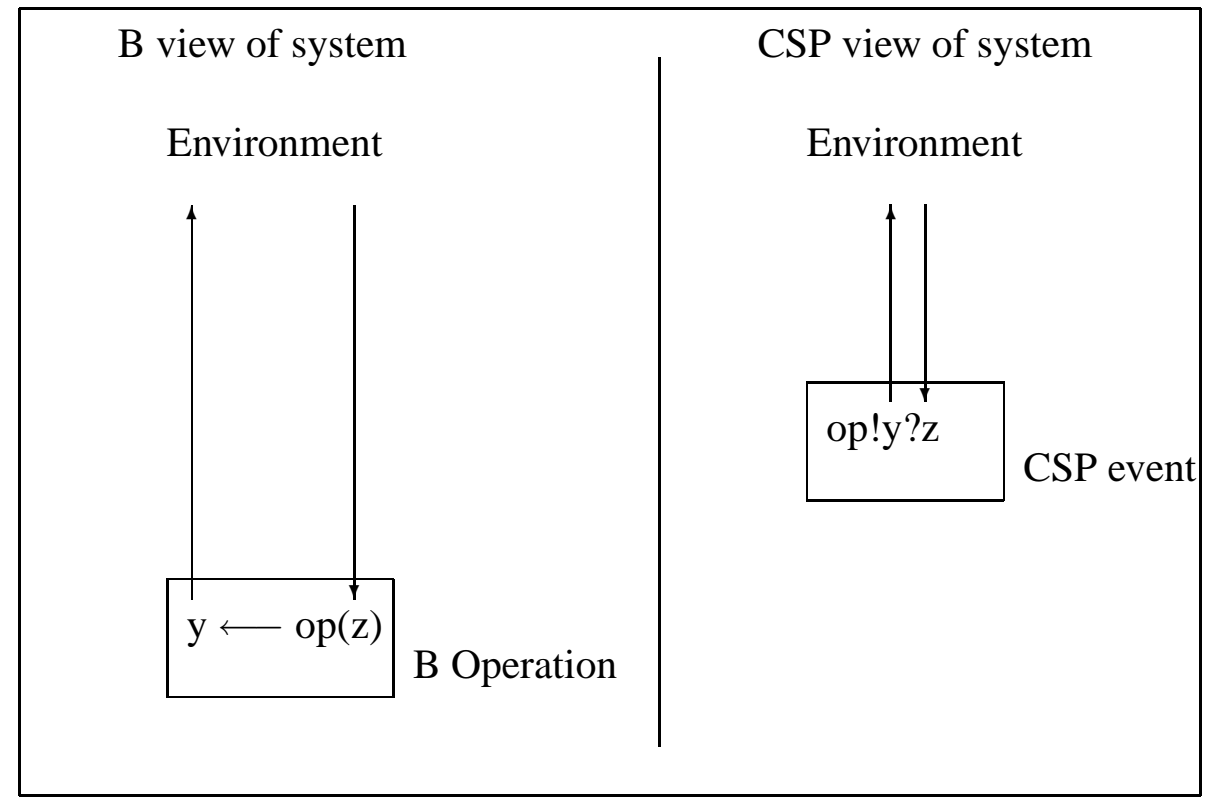

Figure 2. Different views of the same action.

A distinction is drawn between operations that respond to external commands and those that are driven internally. A development will begin with a description of a number of operations: things that the system must do when commanded. During the development refinements will introduce internal operations. We distinguish between external and internal operations by marking the external operations with $/ * e x t * /$ annotations, which are discussed in more detail in the refinement and translation section 5.

Definition 3.6 details the CSP subset of control fragments used in this paper: event prefix, choice, interleaving, if-then-else, and recursion control.

\section{Definition 3.6 (Controller Syntax with I/O)}

$$
\begin{aligned}
R::= & \square_{y} a ! y ? z \rightarrow R \mid \\
& R 1 \square R 2 \mid \\
& \left(\underset{y_{1}}{\square} a_{1} ! y_{1} ? z_{1} \rightarrow \text { skip }|||\ldots||| \square_{y_{n}} a_{n} ! y_{n} ? z_{n} \rightarrow \text { skip } ; R\right) \mid \\
& \square_{y} e ! y \rightarrow \text { if } y \text { then } R 1 \text { else } R 2 \mid \\
& S(p)
\end{aligned}
$$

In this paper the CSP controller is a different view of the annotated B specification. A more complex arrangement arises if the CSP controller is permitted to carry around local state. The simplified view is pictorially represented in figure 2. An annotated B machine output 
is the same as a CSP controller output. In definition 3.6 the channel $a$, in the controller fragment $\square_{y} a ! y ? z \rightarrow R$, is an operation name with a choice over all possible outputs $y$ : from the controller's point of view, if $a$ is called then any output $y$ should be allowed. The outputs are fresh and modelled as a distributed external choice ranging over the type given in the B (the type is not always given in the controller definition). The channel has an input vector $z$. To accommodate analysis, finite types are used in the CSP. The same restriction does not exist in the B. Hence the CSP representation of the B operation may not be a true representation in terms of input and output, which may be a subset of the B types. $S(p)$ is a parameterised process variable. The external choice operator chooses between two process $R 1 \square R 2$ and relates to the /* $O P_{J} \mathrm{NEXT}^{*} /$ annotation that has one set. The interleave operator executes the two or more processes concurrently which will not synchronise on any events. The if - then - else operator makes the decision on $y$; an output of the $e$ operation. Recursive definitions are given as $S \widehat{=} R$. In a controller definition, all process variables used are bound by some recursive definition.

A major constraint is enforced on the way controllers can be written. It facilitates translations, but turns out not to be so troublesome as it first appears. Controllers must start with an initialisation $(R 1)$, then enter a main loop $(S \widehat{=} R 2)$. This is summarized in definition 3.7. A controller $C T R L$ has a definition, $R 1$, given in definition 3.6, in which all the parameterised process variables are the same, $S$. The definition of $S$ is $R 2$ and is also given in definition 3.6. The only recursive calls allowed are to $S$.

\section{Definition 3.7 (Controller Syntax with I/O)}

$$
\begin{aligned}
C T R L= & R 1 \\
S= & R 2 \\
& \text { where } R 1 \text { and } R 2 \text { are terms from definition } 3.6 \text { and } \\
& S \text { is the only recursive variable allowed and } \\
& R 2 \text { is guarded as defined in definition } 3.9
\end{aligned}
$$

The results presented in this paper require that all recursive definitions are guarded, which means that at least one event must occur before a recursive call. The meaning of consistency between the controller and the annotations is given in terms of the init functions. The init function returns a set of operations available next and is developed in definition 3.8

\section{Definition 3.8 (init on CSP controller process with I/O extensions)}

$$
\begin{aligned}
\operatorname{init}\left(\square_{y} a ! y ? z \rightarrow R 1\right) & =\{a\} \\
\operatorname{init}(R 1 \square R 2) & =\operatorname{init}(R 1) \cup \operatorname{init}(R 2) \\
\operatorname{init}\left(\square a_{y_{1}} a_{1} y_{1} ? z_{1} \rightarrow \text { skip }\left.\|\ldots\|\right|_{y_{n}} a_{n} ! y_{n} ? z_{n} \rightarrow \text { skip }\right) ; R & =\left\{a_{1}, a_{2}, \ldots, a_{n}\right\} \\
\text { init }(\text { if y then } R 1 \text { else } R 2) & =\operatorname{init}(R 1) \cup \operatorname{init}(R 2) \\
\operatorname{init}(S(p)) & =\operatorname{init}(R(p))
\end{aligned}
$$

An action prefix must appear with output on the left. In the first case of the init definition the head of the control fragment is extracted. The outputs and inputs of the action are the same as the outputs and inputs of the B operation. The init of a prefixed action is the action (event). 
The init of a choice between two processes is the union of the init of the individual processes. The init of the interleaving is the set of first actions of each interleaving. Annotations clearly show an ordering of operations: an initial operation and a set of next operations. Every operation has a prefix, and is therefore guarded. Every control fragment must have a prefix and hence be guarded. The guard function is defined in definition 3.9. Prefixed operations are guarded. A fragment with an external choice separating the two processes is prefixed if the individual processes are guarded. Similarly with the if-then-else. The parameterised process variable is not guarded, whereas the recursive definition is guarded if the body is guarded.

\section{Definition 3.9 (guarded on CSP controller process with I/O)}

$$
\begin{aligned}
\text { guarded }\left(\square_{y} a ! y ? z \rightarrow R 1\right) & =\text { true } \\
\text { guarded }(R 1 \square R 2) & =\operatorname{guarded}(R 1) \wedge \operatorname{guarded}(R 2) \\
\text { guarded }\left(\left(\square_{y_{1}} a_{1} ! y_{1} ? z_{1} \rightarrow \text { skip } \| \mid\right.\right. & \\
\ldots \mid \| & \\
\left.\left.\square a_{n} ! y_{n} ? z_{n} \rightarrow \text { skip }\right) ; R\right) & =\text { true } \\
\text { guarded }(\text { if } T R U E \text { then } R 1 \text { else } R 2) & =\operatorname{guarded}(R 1) \wedge \operatorname{guarded}(R 2) \\
\text { guarded }(\text { if FALSE then } R 1 \text { else } R 2) & =\operatorname{guarded}(R 1) \wedge \operatorname{guarded}(R 2) \\
\text { guarded }(S(p)) & =\text { false }
\end{aligned}
$$

\section{I/O NEXT Consistency}

Consistency between a guarded controller and the annotated B machine is broken down into initial (definition 4.1) and step-consistency (definition 4.2).

Definition 4.1 (Initial-Consistency of $\mathrm{M}$ with respect to M_CTRL) The initial-consistency of the controller fragment $R$ is defined as follows:

1. $\square_{y} a ! y ? z \rightarrow R$

is initially-consistent with $M$ if $a \in \operatorname{next}($ INITIALISATION) and

$R$ is step-consistent with $M$

2. $R 1 \square R 2$

is initially-consistent with $M$ if $R 1$ and $R 2$ are initially-consistent with $M$.

3. $S(p)$

is initially-consistent with $M$ 
A family of recursive definitions $S \widehat{=} R$ is initially-consistent with $M$ 's annotations if each $R$ is initially-consistent with $M$ 's annotations.

[ We define next $(a)$ as the set of operations in the annotation of a. ]

A controller that starts with an interleaving or a conditional control fragment is not initiallyconsistent and should be avoided. An initialisation can not have an output which rules out the use of an if - then - else annotation on the initialisation. Ruling out the interleaving annotation simplifies initial-consistency checking.

Definition 4.2 (Step-Consistency of $\mathrm{M}$ with respect to M_CTRL) The step-consistency of the controller fragment $R$ is defined as follows:

1. $\square_{y} a ! y ? z \rightarrow R$

is step-consistent with $M$ if $\forall b \bullet b \in \operatorname{init}(R) \Rightarrow b \in \operatorname{next}(a)$, and $R$ is step-consistent with $M$.

2. $R 1 \square R 2$

is step-consistent with $M$ if $R 1$ and $R 2$ are step-consistent with $M$.

3. $\left(\square_{y} a ! y_{a} ? z_{a} \rightarrow\right.$ skip $\| \mid \square_{y} b ! y_{b} ? z_{b} \rightarrow$ skip $) ; R$

is step-consistent with $M$ if $\forall e \bullet e \in \operatorname{init}(R) \Rightarrow e \in \operatorname{next}(a)$ and $e \in \operatorname{next}(b)$, and $R$ is step-consistent with $M$, and update $\left(a ! y_{a} ? z_{a}\right) \cap$ update $\left(b ! y_{b} ? z_{b}\right)=\{\}$.

4. $\square_{y} e \rightarrow$ if $y$ then $R 1$ else $R 2$

is step-consistent with $M$ if $y \in B O O L$ and $R 1$ and $R 2$ are step-consistent with $M$ and

$\forall b \in$ init $(R 1) \Rightarrow b \in$ condition_true $(e)$ and

$\forall c \in$ init $(R 2) \Rightarrow c \in$ condition_false $(e)$

where condition_true returns the actions that are enabled when $y=$ true and condition_false returns the actions that are enabled when $y=$ false.

5. $S(p)$

is step-consistent with $M$

A family of recursive definitions $S \widehat{=} R$ is step-consistent with $M$ 's annotations if each $R$ is step-consistent with M's annotations.

The interleaving operator can only be shown to be consistent in a very limited sense. Two actions are allowed to occur in parallel provided they do not attempt to change the variables used by the other action. 
Definition 4.3 (Consistency) A controller $R$ is consistent with the annotations of machine $M$ if it is step-consistent with M's annotations and initially-consistent with M's annotations.

The main result of this section is that if $R$ is consistent with the annotations of a machine $M$, and the annotations of $M$ are consistent with machine $M$, then operations of $M$ called in accordance with the control flow of $R$ will never be called outside their preconditions. We have [9] proven a theorem that shows that this holds for the basic NEXT, and the NEXT_COND annotations. The annotations are lose enough to permit a large set of possible consistent controllers. As such the controller is viewed as a trace refinement of the annotations. The controllers do not refine the annotations in a failures divergence sense. We believe, but have not yet proven, that the NEXT_PAR and NEXT_SEQ can be rewritten in the basic NEXT form.

The key feature of the proof of this main result is an argument that no trace of $R$ leads to an operation of $M$ called outside its precondition or guard. This is established by building up the traces of $R$ and showing that at each step an operation called outside its precondition cannot be introduced, by appealing to the relevant annotation and applying its proof obligation.

The benefit of this main result is that the details of the operations of $M$ are required only for checking the consistency of the annotations, and are not considered directly in conjunction with the controller. The annotations are then checked against the controller using the definition of consistency above. This enables a separation of concerns, treating the annotations as an abstraction of the B machine.

\section{Refinement and Translation to Handel-C}

Refining should be considered where an otherwise cumbersome translation would result. Narrowing down the choice of the next operation reduces the size of the implementation, and avoids the translation process making an arbitrary choice to resolve the choice in the annotations. The first set of refinements, given in the table in figure 3 replace annotated sets with their subsets: non-determinism is reduced. The operation, like $O P_{J}$, quoted in the table are all sets.

NEXT external choice refinement reduces the non-determinism in the choices that are offered in the next step. The NEXT interleave refinement reduces the non-determinism in one or more branches of the interleave execution. The NEXT sequential refinement reduces the non-determinism in one or more sections of the sequence. The NEXT conditional refinement reduces choice in a similar way.

The second refinement table given in figure 4 outlines some algorithmic refinements. In case 1 a new set of operations are introduced $O P_{J}$. New operations can be introduced into Event-B in subsequent refinements. In traditional B new operations must be introduced beforehand as operators that implement skip. Case 1 refines a simple NEXT operation into a sequence of detailed operations. The refinement sequence must end in the original next operation, which signifies the end of the refinement chain. In case 2 a next sequence NEXT_SEQ to next interleave refinement NEXT_PAR is depicted. It is possible if the operations that would make up the sequence are independent: they neither read nor write to similar variables.

A translations guide for annotations is given in the the table in figure 5 and figure 6 . This is a guide because without the knowledge of the control structure, in particular the points of recursion, a translation can not be automated. However, the annotations do differentiate between internal and external B operations, which has an impact on the final structure of the code. The CSP controller is required to get a full picture for translation and the table in figure 16 and 


\begin{tabular}{|c|c|c|c|}
\hline & Annotation & Refinement & type \\
\hline 1 & $O P_{i}=\ldots O P_{J} N E X T$ & $O P_{i} \widehat{=} \ldots O P_{J}^{\prime} N E X T$ & $\begin{array}{l}\text { next } \\
\text { external } \\
\text { choice } \\
\text { refinement }\end{array}$ \\
\hline 2 & $\begin{array}{l}O P_{i} \widehat{=} \ldots O P_{J} O P_{K} N E X T \_P A R \\
O P_{j_{1}} \widehat{=} \ldots O P_{X} N E X T \\
\cdots \\
O P_{j_{n}} \widehat{=} \ldots O P_{X} N E X T \\
O P_{k 1} \widehat{=} \ldots O P_{X} N E X T \\
\ldots \\
O P_{k n} \widehat{=} \ldots O P_{X} N E X T\end{array}$ & $\begin{array}{l}O P_{i}=\ldots O P_{J}^{\prime} O P_{K}^{\prime} N E X T \_P A R \\
O P_{j_{1}} \widehat{=} \ldots O P_{X} N E X T \\
\ldots \\
O P_{j_{n}} \widehat{=} \ldots O P_{X} N E X T \\
O P_{k 1} \widehat{=} \ldots O P_{X} N E X T \\
\ldots \\
O P_{k n} \widehat{=} \ldots O P_{X} N E X T\end{array}$ & $\begin{array}{l}\text { next } \\
\text { interleave } \\
\text { refinement }\end{array}$ \\
\hline 3 & $\begin{array}{l}O P_{i} \widehat{=} \ldots O P_{J} O P_{P} N E X T \_S E Q \\
O P_{j_{1}} \widehat{=} \ldots O P_{P} N E X T \\
\cdots \\
O P_{j_{n}} \widehat{=} \ldots O P_{P} N E X T\end{array}$ & $\begin{array}{l}O P_{i} \widehat{=} \ldots O P_{J}^{\prime} O P_{P}^{\prime} N E X T \_S E Q \\
O P_{j_{1}} \widehat{=} \ldots O P_{P} N E X T \\
\cdots \\
O P_{j_{n}} \widehat{=} \ldots O P_{P} N E X T\end{array}$ & $\begin{array}{l}\text { next } \\
\text { sequential } \\
\text { refinement }\end{array}$ \\
\hline 4 & $\begin{array}{l}O P_{i} \widehat{=} \ldots O P_{J} O P_{P} N E X T \_C O N D \\
O P_{j_{1}} \widehat{=} \ldots O P_{P} N E X T \\
\ldots \\
O P_{j_{n}} \widehat{=} \ldots O P_{P} N E X T\end{array}$ & $\begin{array}{l}O P_{i} \widehat{=} \ldots O P_{J}^{\prime} O P_{P}^{\prime} N E X \_C O N D \\
O P_{j_{1}} \widehat{=} \ldots O P_{P} N E X T \\
\ldots \\
O P_{j_{n}} \widehat{=} \ldots O P_{P} N E X T\end{array}$ & $\begin{array}{l}\text { next } \\
\text { condition } \\
\text { refinement }\end{array}$ \\
\hline & & $\begin{array}{c}\text { where } O P_{J}^{\prime} \subseteq O P_{J} \text { and } \\
\qquad O P_{K}^{\prime} \subseteq O P_{K}\end{array}$ & \\
\hline
\end{tabular}

Figure 3. NEXT Refinements - Reduction of Non-determinism.

to some extent the table in figure 1 illustrates how translation of the control can proceed. As mentioned, the translation of a particular annotated operator is dependent on whether the operation is an internal or external operation. Internal operations can execute immediately after invocation. The execution of an external operation must wait for external stimulus: a change in the command input bus. A wait loop is introduced to poll the appropriate input bus until an external operation invocation is detected: wait_on_.... Some annotated operators have restrictions on their I/O mode. External operators are marked with / $*$ ext $* /$. The NEXT_PAR can only be associated with internal operations next. The NEXT_SEQ must have an external operator at the head of the sequence and internal operations following. This restriction relates to the way this annotation is used in refinement. The CSP controller does not differentiate between internal and external operations. Hence the tables in figures 5, figure 6, 15, 16, and 


\begin{tabular}{|c|c|c|c|}
\hline & Annotation & Refinement & type \\
\hline 1 & $O P_{i} \widehat{=} \ldots O P_{X} N E X T$ & $\begin{array}{l}O P_{i} \widehat{=} \ldots O P_{J} O P_{X} N E X T \_S E Q \\
O P_{j_{1}} \widehat{=} \ldots O P_{X} N E X T \\
O P_{j_{n}} \widehat{=} \ldots O P_{X} N E X T\end{array}$ & $\begin{array}{l}\text { introduction } \\
\text { of } \\
\text { new } \\
\text { operation }\end{array}$ \\
\hline 2 & $\begin{array}{l}O P_{i} \widehat{=} \ldots O P_{J} O P_{P} N E X T \_S E Q \\
O P_{j_{1}} \widehat{=} \ldots O P_{P} N E X T \\
\ldots \\
O P_{j_{n}} \widehat{=} \ldots O P_{P} N E X T \\
\text { variable_used }\left(\left\{O P_{j}, \ldots, O P_{k}\right\}\right) \\
\cap \\
\text { variable_used }\left(\left\{O p_{p}, \ldots, O P_{q}\right\}\right) \\
=\{\}\end{array}$ & $\begin{array}{l}O P_{i} \widehat{=} \ldots O P_{J} O P_{P} N E X T \_P A R \\
O P_{j_{1}} \widehat{=} \ldots O P_{P} N E X T \\
\ldots \\
O P_{j_{n}} \widehat{=} \ldots O P_{P} N E X T\end{array}$ & $\begin{array}{l}\text { next } \\
\text { sequence } \\
\text { to } \\
\text { interleave } \\
\text { refinement }\end{array}$ \\
\hline & & & \\
\hline
\end{tabular}

Figure 4. NEXT Refinements - Structural Refinements.

1 are all required to obtain a translation.

In the table in figure 5 and figure 6 NEXT a annotation with one next operation translates to a sequence of two operations. If the second operation is an internal operation then it is case 1: all its inputs are not ported. If the second operation is an external operation (all inputs are ported) then case 2 is the translation template. The controller will wait until a new command arrives then execute the external operation if it was requested. Case 3 , sequential arrangement of external operations, is restricted to external operations only. A translation of a sequence that starts with one operation then has a choice of several external operations will test each input set and execute the first operation for which the input has change since its last execution. (The new input values must be latched in.) Interleave action is only permitted between internal operations (case 4): those that take their input from internal variables. The Handel-C par statement ensures that all the branches when complete wait until the longest (in terms of clock cycles) has completed. The conditional operator can be used for internal or external action. In the table in figure 6 case 5 is the translation of the NEXT_SEQ. In the previous section the NEXT_SEQ was introduced to support refinement: a basic NEXT is refined into a sequence of operations NEXT_SEQ. To refine an operation that both inputs and outputs, a sequence of operations must input at the beginning of the sequence and output at the end of the sequence. Case 5 reflects this requirement: the first operation in the sequence is an external operation that inputs and the final operation is an internal operation that outputs.

The translations of Stepney [16], and Phillips and Stilles [12] are given in table 1. Only the translation of parametrisable integer declaration, functions, and recursion are used. This is because our source is not CSP (it is annotated B and CSP) as such channels are not being used to synchronise events. In the table the CSP language construct and translation are mapped. A tick is inserted if they are supported by Stepney (SS) or Phillips and Stilles (PS). When an operation is invoked it takes its input from the environment from the port. Internal synchronisation of operations within machines is not dealt with in this paper. To guide the B translation the table in figure 15 has been developed. A discussion of the example is given in 


\begin{tabular}{|c|c|c|c|}
\hline & Annotation & Handel-C Translation Fragment & Comment \\
\hline 1 & $\begin{array}{l}O P_{i} \widehat{=} \ldots\left\{O P_{j_{1}}\right\} N E X T \\
o p_{i} ! y_{i} ? z_{i} \rightarrow\left(o p_{j_{1}} ! y_{j_{1}} ? z_{j_{1}} \rightarrow \ldots\right.\end{array}$ & $y_{i}=O P_{i}\left(v_{i}\right) ; y_{j_{1}}=O P_{j_{1}}\left(v_{j_{1}}\right)$ & $\begin{array}{l}\text { internal } \\
\text { single next } \\
\text { translation }\end{array}$ \\
\hline 2 & $\begin{array}{l}O P_{i} \widehat{=} \ldots\left\{O P_{j_{1}}\right\} N E X T \\
/ * \text { ext } * / O P_{j_{1}} \widehat{=} \ldots \\
o p_{i} ! y_{i} ? z_{i} \rightarrow\left(o p_{j_{1}} ! y_{j_{1}} ? z_{j_{1}} \rightarrow \ldots\right.\end{array}$ & $\begin{array}{l}y_{i}=O P_{i}\left(v_{i}\right) \\
\text { wait_on_OP } \\
\text { if in }=O P_{j_{1}} \\
\left.\text { then } y_{j_{1}}=O P_{j_{1}}\left(v_{j_{1}}\right)\right\} \\
\text { else delay; }\end{array}$ & $\begin{array}{l}\text { external } \\
\text { single next } \\
\text { translation }\end{array}$ \\
\hline 3 & $\begin{array}{c}/ * e x t * / O P_{i} \widehat{=} \ldots \\
\left\{O P_{j_{1}}, \ldots, O P_{j_{n}}\right\} N E X T \\
o p_{i} ! y_{i} ? z_{i} \rightarrow\left(o p_{j_{1}} ! y_{j_{1}} ? z_{j_{1}} \rightarrow \ldots \square .\right. \\
\square \\
\left.o p_{j_{n}} ! y_{j_{n}} ? z_{j_{n}} \rightarrow \ldots\right)\end{array}$ & $\begin{array}{l}y_{i}=O P_{i}\left(z_{i}\right) \\
\text { wait_on_OP } \\
\text { if in }=O P_{j_{1}}-\cdots-O P_{j_{n}} \\
\text { then } y_{j_{1}}=O P_{j_{1}}\left(v_{j_{1}}\right) \\
\text { else } \ldots \\
\quad \ldots \\
\text { if in }=O P_{j_{n}} \\
\text { then } y_{j_{n}}=O P_{j_{n}}\left(v_{j_{n}}\right) \\
\text { else skip }\end{array}$ & $\begin{array}{l}\text { external } \\
\text { multiple } \\
\text { next } \\
\text { choice } \\
\text { translation }\end{array}$ \\
\hline 4 & $\begin{array}{l}O P_{i}=\ldots O P_{j} O P_{k} N E X T \_P A R \\
O P_{j}=\ldots O P_{X} N E X T \\
O P_{k} \widehat{=} \ldots O P_{X} N E X T \\
o p_{i} ! y_{i} ? z_{i} \rightarrow\left(o p_{j} ! y_{j} ? z_{j} \rightarrow \ldots\right) \| \\
\quad\left(o p_{k} ! y_{k} ? z_{k} \rightarrow \ldots\right)\end{array}$ & $\begin{array}{c}\operatorname{seq}\left\{y_{i}=O P_{i}\left(v_{i}\right)\right. \\
\operatorname{par}\left\{y_{j}=O P_{j}\left(v_{j}\right)\right. \\
y_{k}=O P_{k}\left(v_{k}\right) \\
\} \\
\}\end{array}$ & $\begin{array}{l}\text { internal } \\
\text { next } \\
\text { interleave } \\
\text { translation }\end{array}$ \\
\hline
\end{tabular}

Figure 5. NEXT Annotation Translation Guide Part 1.

section 6.

\section{Example: Safe Control System}

We use the example of a safe locking system to illustrate the ideas introduced in the previous sections. The abstract specification outlines the operations of the environment. The operations that are invoked by the environment are indicated with $/ *$ ext $* /$ annotations. Both the operation output and the operation can be marked with / $*$ ext $* /$ annotations. All $/ * e x t * /$ annotation outputs are ported and become part of the Handel-C interface output. All $/ *$ ext $* /$ operations are associated with a bus port that has a state of the same name as the operation. Variables intended as input are marked with $/ * I N * /$. It is possible to mark the variables as 


\begin{tabular}{|c|c|c|c|}
\hline & Annotation & Handel-C Translation Fragment & Comment \\
\hline 5 & 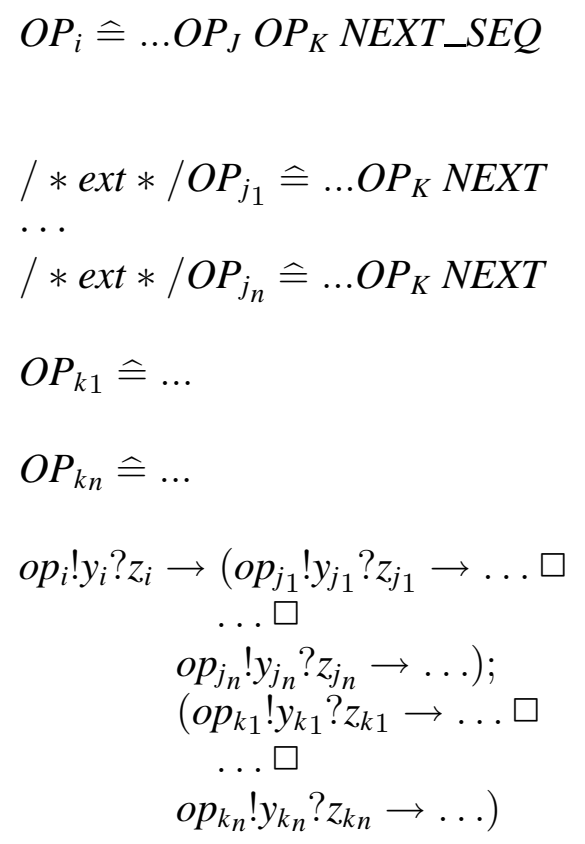 & $\begin{array}{l}y_{i}=O P_{i}\left(v_{i}\right), \text { wait_on_OP } \\
\text { if in }=O P_{j_{1}} \\
\text { then } y_{j_{1}}=O P_{j_{1}}\left(v_{j_{1}}\right) \\
\text { else } \ldots \\
\quad \ldots \\
\text { if in }=O P_{j_{n}} \\
\text { then } y_{j_{n}}=O P_{j_{n}}\left(v_{j_{n}}\right) \\
\text { else skip } \\
\quad ; \\
y_{k 1}=O P_{k 1}\left(v_{k 1}\right)\end{array}$ & $\begin{array}{l}\text { next } \\
\text { sequential } \\
\text { translation }\end{array}$ \\
\hline 6 & 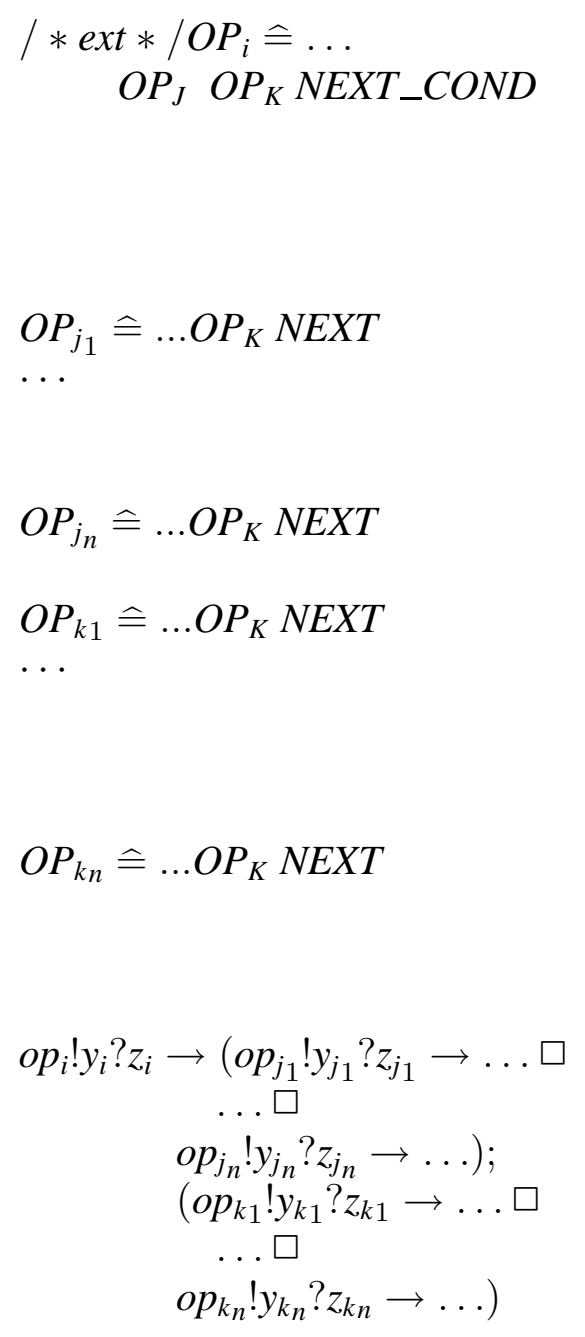 & $\begin{array}{l}y=O P_{i}\left(v_{i}\right), \\
\text { if } y \\
\{\text { wait_on_OP } \\
\text { if in }=O P_{j_{1}} \\
\text { then } y_{j_{1}}=O P_{j_{1}}\left(v_{j_{1}}\right) \\
\text { else } \ldots \\
\ldots \\
\text { if in }=O P_{j_{n}} \\
\text { then } y_{j_{n}}=O P_{j_{n}}\left(v_{j_{n}}\right) \\
\text { else skip } \\
\} \\
\text { else } \\
\text { \{wait, } \\
\text { if in }=O P_{k 1} \\
\text { then } y_{k 1}=O P_{k 1}\left(v_{k 1}\right) \\
\text { else } \ldots \\
\quad \ldots \\
\text { if in }=O P_{k n} \\
\text { then } y_{k n}=O P_{k n}\left(v_{k n}\right) \\
\text { else skip } \\
\text { \} }\end{array}$ & $\begin{array}{l}\text { external } \\
\text { next } \\
\text { condition } \\
\text { translation }\end{array}$ \\
\hline
\end{tabular}

Figure 6. NEXT Annotation Translation Guide Part 2. 
Table 1. Existing CSP to Handel-C Translation Guide.

\begin{tabular}{|c|c|c|c|c|}
\hline Feature & CSPM & Handel-C & PS & SS \\
\hline $\begin{array}{l}\text { Channel Declarations } \\
\text { (from use) }\end{array}$ & channel & chan, chanin, chanout & $\bar{\nabla}$ & \\
\hline Channel Declarations & channel c & chan SYNC c; & & $\checkmark$ \\
\hline $\begin{array}{l}\text { Typed Structured } \\
\text { Channel Declarations }\end{array}$ & channel d: T.T & chan struct d_DATA d & & $\bar{\checkmark}$ \\
\hline Input Channel Operations & in? $x$ & in?x; & $\checkmark$ & $\checkmark$ \\
\hline Output Channel Operations & out!x & out!x & $\checkmark$ & $\checkmark$ \\
\hline Integer Declarations & & int $8 x$ & $\checkmark$ & $\checkmark$ \\
\hline Parametrisable functions & $\mathrm{p}(\mathrm{n})=\ldots$ & $\operatorname{void}(n) \ldots$ & $\checkmark$ & $\bar{\checkmark}$ \\
\hline External Choice & [ ] & prialt ... & $\checkmark$ & $\bar{\checkmark}$ \\
\hline Synchronous Parallel & {$[|\{|\ldots|\}|]$} & par ... & $\checkmark$ & $\checkmark$ \\
\hline Replicated Sharing Parallel & {$[\mid$ Event $\mid]$ n: $\{$ i..j $\} \bullet P(n)$} & $\operatorname{par}\left(n=\mathrm{i} ; \mathrm{n}_{\mathrm{i}}=\mathrm{j} ;++\mathrm{n}\right) \mathrm{P}(\mathrm{n})$ & & $\checkmark$ \\
\hline Recursion & $\mathrm{P}=\ldots \rightarrow \mathrm{P}$ & while(1) ... & $\checkmark$ & $\checkmark$ \\
\hline Conditional Choice & if $\mathrm{b}$ then $\mathrm{P}$ else $\mathrm{Q}$ & if (B) then $\mathrm{P}()$; else $\mathrm{Q}()$; & & $\checkmark$ \\
\hline Macros & $\{-\ldots-\}$ & $\ldots$ & $\checkmark$ & \\
\hline
\end{tabular}

$/ * I N * /$ or $/ * O U T * /$. Along with the mode the width of the type is given in bits. Operations are invoked in two ways. The first way has already been introduced; an / * ext $* /$ operation will have a input bus associated with it, which when set to the operator name will invoke the operation when it is enabled by the control flow. Operations not labelled with $/ *$ ext $* /$ are internal and are invoked immediately when enabled by the control flow.

\subsection{The Example's State and Control Flow}

In figure 7 the B Abstract Machine for the safe is given. There are three command states Locked, Unlocked, and BrokenOpen which are represented in two bits. The variable Door is drawn from the COMMAND type and initialised to Unlocked. The Lock operation is enabled after initialisation. It is an external operation with externally ported output. After setting the Door state variable to Locked, Unlocked and BreakOpen are enabled. For completeness we introduce two operations that will be used later to develop the detailed functionality of the machine during refinement. These operations are UnlockR 1 and UnlockR2. Their bodies are not expanded. The Unlock is an external operation and has externally ported output. It nondeterministically decides to set the Door variable to Unlocked or Locked. The next operator to be enabled depends on the outcome of the Unlock operation. If Unlocked was chosen then the next enabled operation is Lock, otherwise Unlocked or BreakOpen will be offered. The BreakOpen operation sets the Door state to BrokenOpen and offers itself as the next operation available.

The controller CTRL given in figure 8 first performs a Initialisation then a Lock and then jumps to the $S$ process where it can perform either an Unlock or BreakOpen. The Unlock event has a single output that is used as the conditional test in the if-then-else following the Unlock event. If the output of the Unlock operation is true then the flow of control is repeated starting again at $C T R L$, if it is false then control is repeated at $S$ 
MACHINE Safe

SETS COMMAND $=\{$ Locked, Unlocked, BrokenOpen $\} / * 2 * /$

VARIABLES Door

INVARIANT Door $\in$ COMMAND $/ *$ OUT2 $* /$

INITIALISATION Door $:=$ Unlocked $\quad / *\{$ Lock $\}$ NEXT */

OPERATIONS

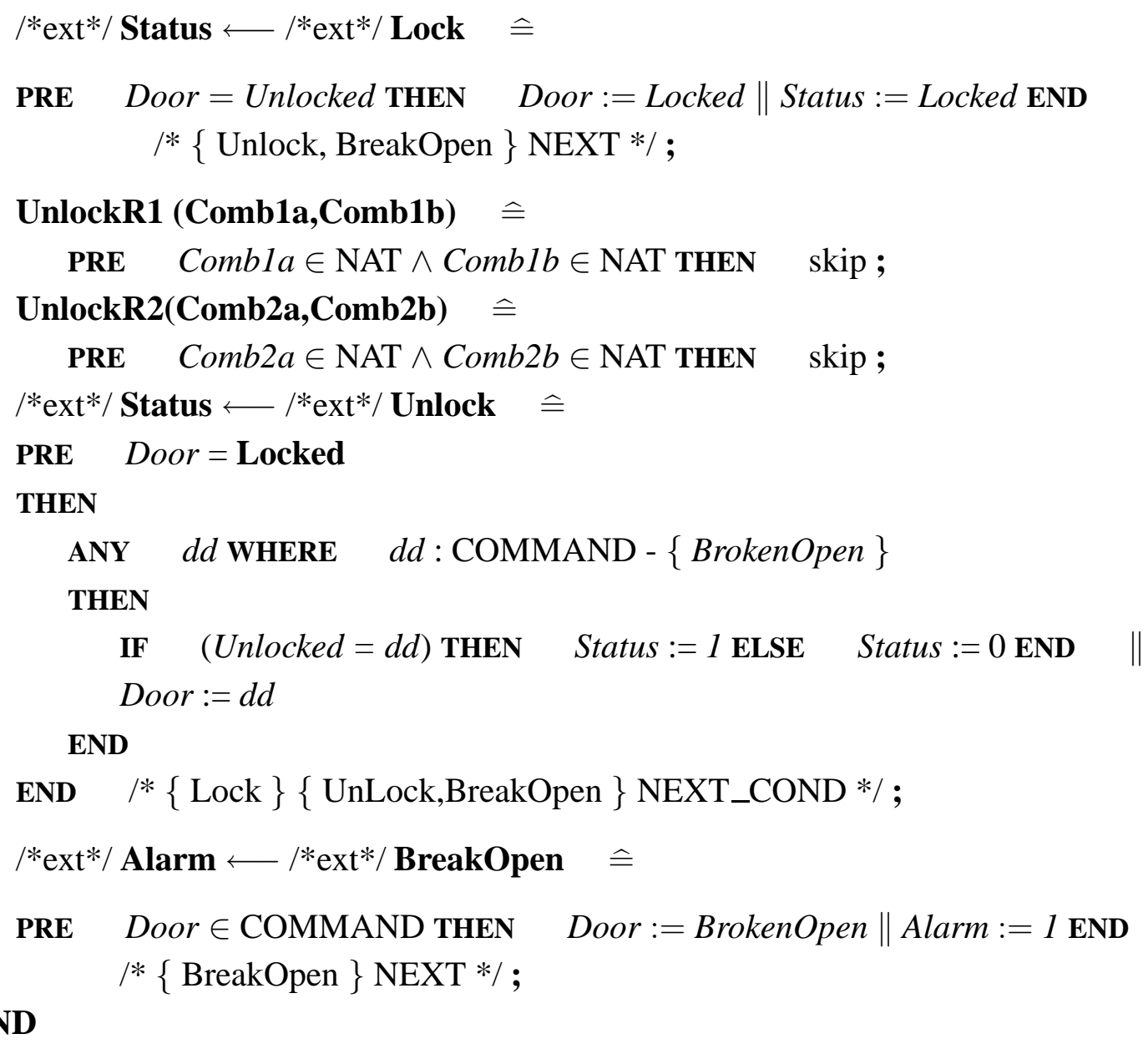

Figure 7. Safe Machine

\subsection{A Refined Example}

A refinement of the Safe machine, called SafeR, is given in figure 9 and figure 10 . It is, within a B framework, mimicking refinement in Event-B. The operation UnlockR 1 and UnlockR 1 are introduced to refine Unlock. The laws of refinement of Event-B are not fully justified. The refined Safe REFINEMENT, SafeR, breaks down the Unlocking process into two stages. Firstly, a two new operation are slotted into the control in parallel: UnlockR1(Comb1a,Comb1b) and UnlockR2(Comb2a,Comb2b). Both have a combination parameter which is compared against a stored master code and a secondly parameter that is used to create a new master key. The UnlockR commands update the master combination if a sucessful comparison occurs. New input variables are added: $C x 1 a, C x 2 a, C x 1 b$, and $C x 2 b$. These are used to input the combination values and are not used by the B Operations. Checked1, Checked2, Master 1 and Master 2 are new variables used by the operations. The annotations of the Lock operation are 


$$
\begin{aligned}
& C T R L=\text { Initialisation } \rightarrow \underset{y}{\square} \text { Lock } ! y \rightarrow S \\
& S \bumpeq(\underset{y}{\square} \text { Unlock!y } \rightarrow(\text { if y then } \underset{y}{\square} \text { Lock } ! y \rightarrow \text { CTRL else } S)) \square \\
& (\underset{y}{\square} \text { BreakOpen!y } \rightarrow \text { B_CTRL) } \\
& B \_C T R L=\square_{y} \text { BreakOpen!y } \rightarrow \text { B_CTRL }
\end{aligned}
$$

Figure 8. Safe Machine Controller.

refined. Two operation are added before the Unlock. The extra proof obligations can be discharged. The bodies of the UnlockR and Rekey(Comb2) are completed at this level. The body of the Unlock operation is refined. The annotations of the Unlock are refined: the BreakOpen operation is removed as an option. What was one unlock operation has been expanded into three (two inparallel). Before refinement the Unlock operation has both input and output. The refined version has the input occuring on the first operations in the refined sequence of operations (UnlockR1 and UnlockR2), and the output occuring on the final operation of the sequence (the original Unlock operation).

The controller given in figure 11 starts off like the abstract process with an Initialisation and a Lock then a jump to $S$. There is in this refined process no choice to breakOpen, only UnlockR1and UnlockR2 are offered with $C x 1 a$ and $C x 1 b$ and $C x 2 a$ and $C x 2 b$ are offered as an input,respectively. The UnlockR process is the first in a sequence of processes that refines the original UnLock process. The refined sequence starts with a parallel combination of UnlockR1 and UnlockR2 events then the original Unlock event, at which point the output is given. As before the outcome of Unlock determines what happens next. If the Unlock was successful the process will be restarted from the beginning. If the current attempt at locking failed then another go at Unlock will occur. It is noted that the Lock $\rightarrow S$ could have been replaced by $C T R L$. However, the former is easier to translate.

\subsection{A Hand Translation using the Guidance}

A summary of the hand translations on the refined B specification is given in the table in figure 15. The B provides the details of the types, variables, and functions. The CSP controller provides the executions details that are use later to construct the Handle-C main section. A summary of the hand translation of the controller is given in the table in figure 16

First we review the B translation. The SETS clause is translated into an enumerated type. The INVARIANT section is used to create the declarations. Variables annotated with a mode will be created as buses of the appropriate I/O type and size. Other variables will be created. Variables which will be bound to ports are created. Each operation which is external is associated with a command input bus of the same name as the machine. The mechanism for requesting an external operation to execute is to change the data on the command input bus to the same name as the operation required. The last requested operation is latched into variable of the same name as the refined machine with a ._var post fix. Variables are declared for operation outputs. The names of the output bus variables are a concatenation of the operation output name and the operation name. This avoids clashes with similar operation output names. Buses are defined for each $/ * I N * /$ and $/ * O U T * /$ annotation, external operation, and operation output. Each operation is translated into a function. If an operation has an output the function will return a value. Functions with outputs will have an assign- 
REFINEMENT SafeR

REFINES Safe

VARIABLES Door, $C x 1 a, C x 2 a, C x 1 b, C x 2 b$, Master1, Checked1 Master2, Checked2

INVARIANT

$C x l a \in \mathrm{NAT} / * \mathrm{IN} 16 * / \wedge C x 2 a \in \mathrm{NAT} / * \mathrm{IN} 16 * / \wedge$

$C x 1 b \in \mathrm{NAT} / * \mathrm{IN} 16 * / \wedge C x 2 b \in \mathrm{NAT} / * \mathrm{IN} 16 * / \wedge$

Master $1 \in \mathrm{NAT} / * 16 * / \wedge$ Checked $1 \in \mathrm{NAT} / * 1 * / \wedge$

Master $2 \in \mathrm{NAT} / * 16 * / \wedge$ Checked $2 \in \mathrm{NAT} / * 1 * /$

\section{INITIALISATION}

Door:=unlocked || Cx1a: $=0\|C x 2 a:=0|| C x 1 b:=0|| C x 2 b:=0\|$

Master $1:=67||$ Checked1 $:=0 \quad$ Master $2:=76||$ Checked $2:=0 \quad \mid *\{$ Lock $\}$ NEXT */

\section{OPERATIONS}

$/ * \operatorname{ext} 2 * /$ Status $\longleftarrow / * \operatorname{ext} 1 * /$ Lock $\hat{=}$

PRE

Door $=$ Unlocked

THEN

Door $:=$ Locked $\|$ Status $:=$ Locked $\|$ Checked $1:=\mathbf{0} \|$ Checked $2:=\mathbf{0}$

END

/* $\{$ UnlockR1(Cx1a,Cx1b), UnlockR1(Cx2a,Cx2b) $\}\{$ Unlock $\}$ NEXT_SEQ */

/* $\{$ UnlockR1(Cx1a,Cx1b) $\}\{$ UnlockR1(Cx2a,Cx2b) $\}$ NEXT_PAR */;

$/ * \operatorname{ext} 1 * /$ UnlockR1(/*16*/Comb1a,/*16*/Comb1b) $\hat{=}$

PRE

Door $=$ Locked

THEN

IF

$($ Comb1a $=$ Master 1$)$

THEN

Checked $1:=\mathbf{1}||$ Master $1:=$ Comb1b

ELSE

Checked $1:=\mathbf{0}$

END

END $\quad / *\{$ Unlock $\}$ NEXT $* /$;

Figure 9. Safe Refinement Part 1.

ment in them that assigns to the bus output function variable. The function will also return that output in the final statement of the function. Assigning to the function output variable and writing it to a output port as well allows it to be put out on the output bus, and used internally in the Handel-C program. The bodies are translated in a straightforward manner. Assignments in the operations are put together in a par Handel-C statement. Assignment and 
PRE

Door $=$ Locked

THEN

IF

$($ Comb2a $=$ Master2 $)$

THEN

Checked $2:=\mathbf{1}||$ Master $2:=\mathbf{C o m b 2 b}$

ELSE

Checked $2:=\mathbf{0}$

END

END $/ *\{$ Unlock $\}$ NEXT */;

$/ * \operatorname{ext} 2 * /$ Status $\longleftarrow$ Unlock $\hat{=}$

PRE

Door $=$ Locked

THEN

Status := Checked \|

IF

$($ Checked $1=1) \wedge($ Checked $2=1)$

THEN

Door := Unlocked

ELSE

Door := Locked

END

END $/ *\{$ Lock $\}\{$ UnlockR $\}$ COND_NEXT */;

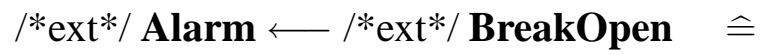

PRE Door $\in$ COMMAND THEN Door $:=$ BrokenOpen $\|$ Alarm $:=1$ END

/* $\{$ BreakOpen $\}$ NEXT */

END

Figure 10. Safe Refinement Part 2

$$
\begin{aligned}
C T R L= & \text { Initialisation } \rightarrow \underset{y}{\square} \text { Lock } ! y \rightarrow S \\
S= & (\text { UnlockR } 1 ? \text { Cx } 1 a ? C x 1 b \rightarrow \text { skip } \| \text { UnlockR } 1 ? \text { Cx } 2 a ? C x 2 b \rightarrow \text { skip }) \rightarrow \\
& \square \text { Unlock!y } \rightarrow\left(\text { if } y \text { then } \square_{y} \text { Lock } ! y \rightarrow \text { S else } S\right)
\end{aligned}
$$

Figure 11. Refined Safe Controller.

the if - then - else B constructs have straightforward translations. The refined B example is limited to assignment and if - then-else. The INITIALISATION is translated into a function called Initialisation_fnc.

The CSP controller is used to construct the main Handel-C body. A summary of the hand 
$/ /$ set clock = external "Clock";

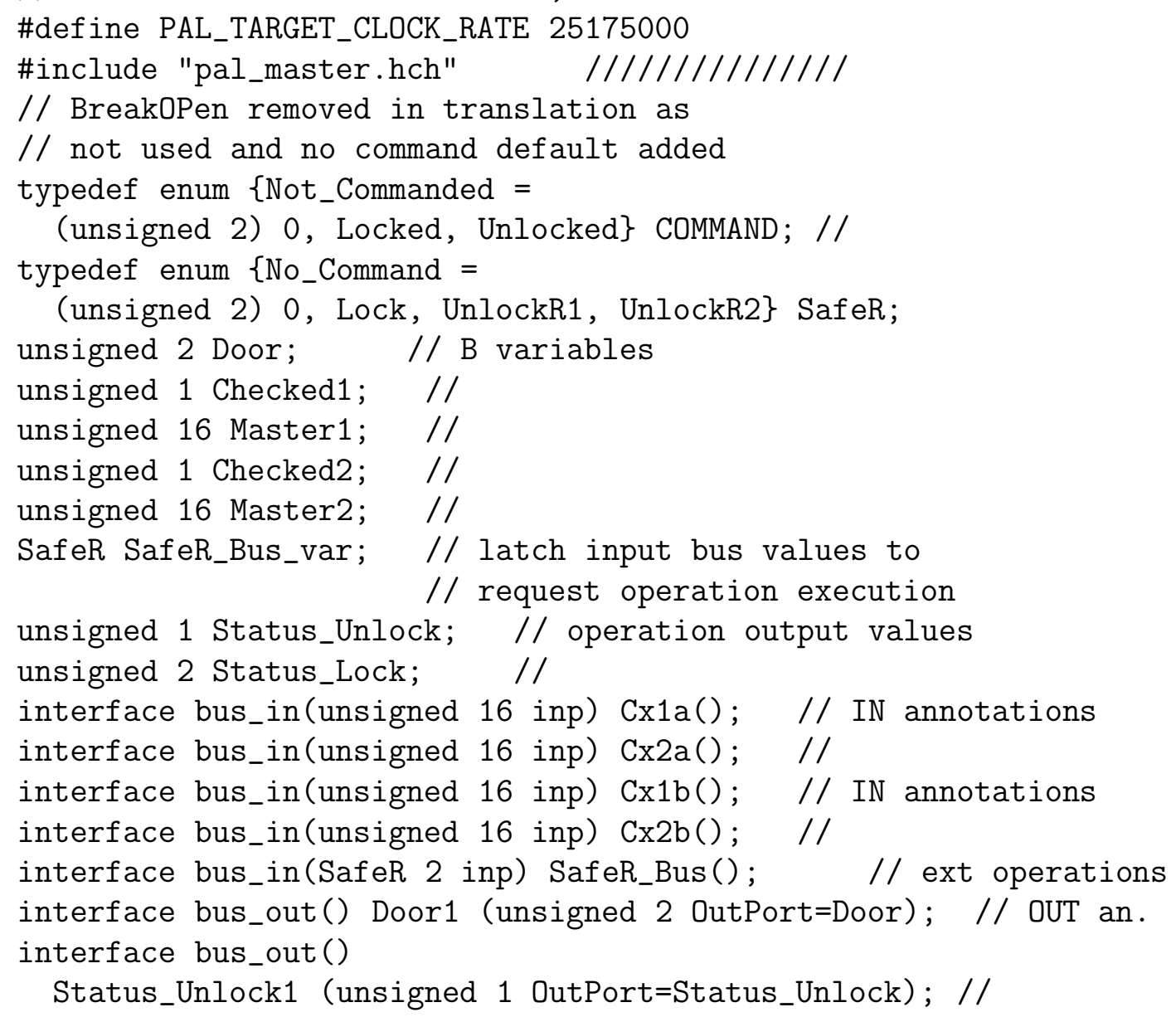

Figure 12. SafeR Translation Part 1a.

translations made on the CSP controller are given in the table in figure 16. The controller design was structurally limited to facilitate translation: initialisation and setting up operations are performed before a main loop is entered. The first process definition CTRL_fnc is not recursive; it is an open process. It translates to a function call CTRL_fnc, which invokes the Initalisation_fnc and lock_fnc functions. On returning to the main program the next function called is the $S \_f n c$, which implements the main loop. $S \_f n c$ is tail recursive and is implemented with a continuously looping while loop; it is a closed process. The first event in the main loop is the UnlockR commands. In the translation the Unlock fnc is preceded by wait_Unlock_fnc as it is an external operation. The UnlockR_fnc functions inputs from the $C x 1 a, C x 1 b, C x 2$, and $C x 2$ input buses. The Unlock_fnc call follows. Unlock_fnc returns a value that is assigned to a variable that is output ported. The value is also used to decide the course of the following if-then-else. Either a Lock_fnc or an UnlockR_fnc is performed after a wait. Then the process recurses.

\section{Discussion}

This paper has introduced a way of refining annotations that support Event-B style refinement, and set out a guide for translation to an HDL, within the B annotation framework. We have demonstrated how the framework previously presented can be extended for both traditional B and Event-B. Our approach sits naturally with refinement. Refinement and translation are still being considered for CSP $\|$ B. In fact the B annotation approach offerers sev- 


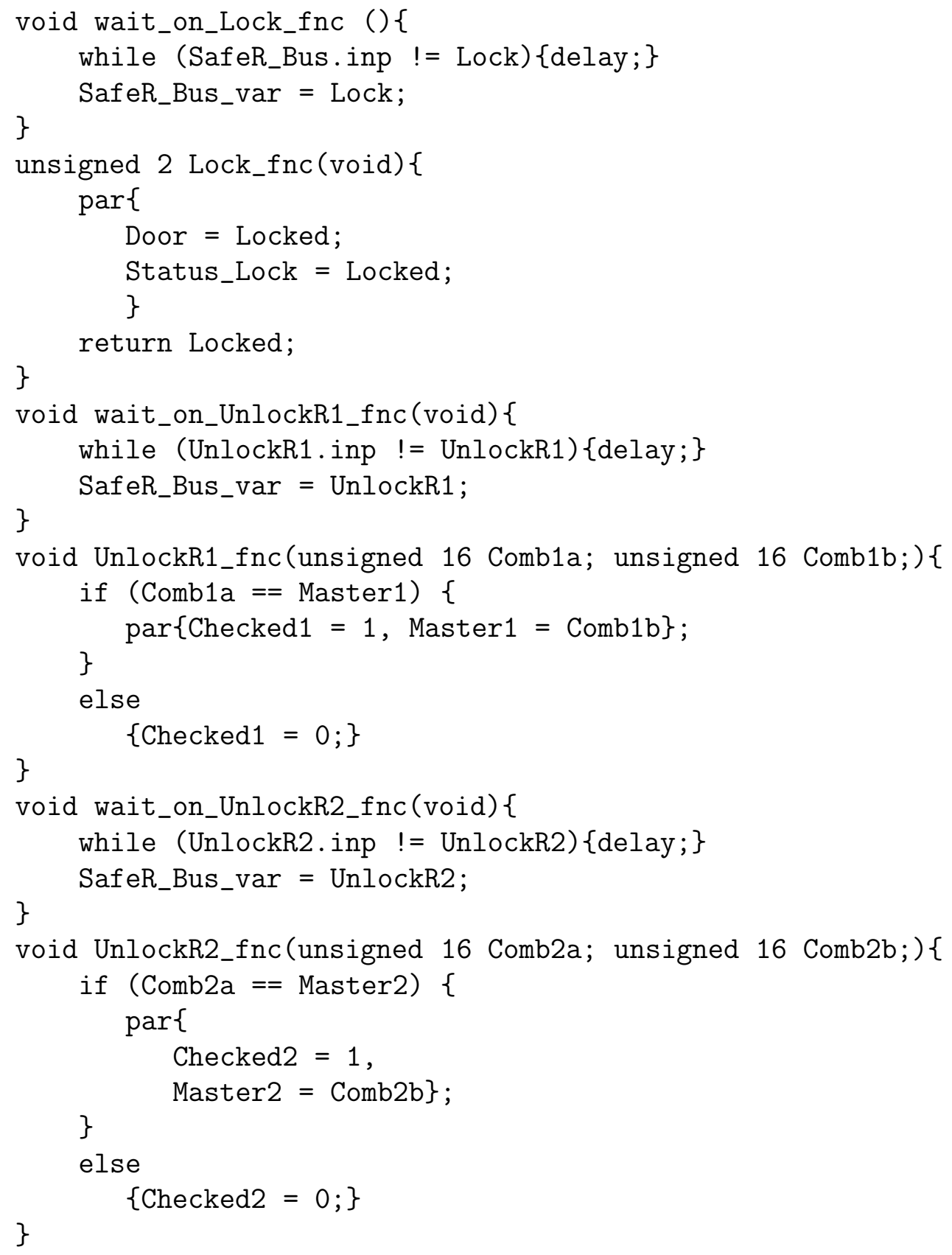

Figure 13. SafeR Translation Part $1 b$.

eral approaches to refinement: refinement of control flow only, state only, or control flow and state. The extensions to the annotations are fairly rich and now include annotations to support: next selection, sequencing, conditional, parallel execution, and I/O. The inability to define points of recursion has led to a reliance on a CSP controller. We restricted this paper to the consideration of fixed variables as operation inputs, and permitted no scope for controller state. Work on CSP state and defining recursive points in the annotations is currently ongoing. More work is required to automate the translation and develop the proof of the theorem to cover interleaving.

\section{Acknowledgements}

The extensions to the refinement have benefited from conversations with Stefan Hallestede and Helen Treharne. Thankyou for the encouraging comments from the refers and detaled 


\section{References}

[1] J-R. Abrial. The B-Book: Assigning Programs to Meaning. Cambridge University Press, 1996.

[2] J-R. Abrial. Event driven circuit construction version 5. MATISSE project, August 2001.

[3] J-R. Abrial and L. Mussat. Event B Reference Manual. ClearSy, 1999.

[4] A. Aljer and P. Devienne. Co-design and refinement for safety critical systems. In 19th IEEE International Symposium on Defect and Fault Tolerance in VSLI Systems (DFT'04), pages 78-86, 2004.

[5] P. T. Ashenden. The Designer's Guide to VHDL. Morgan Kaufmann, 1996.

[6] C. Fischer. CSP-OZ: A combination of Object-Z and CSP.

[7] C. A. Hoare. Communicating Sequential Processes. Prentice-Hall International, Englewood Cliffs, New Jersey, 1985.

[8] W. Ifill. Formal development of an example processor (AEP) in AMN, C and VHDL. Computer science, University of London, Computer Science Department, Royal Holloway, University of London, Egham, Surrey TW20 OEX, Sept 1999.

[9] W. Ifill, S. Schneider, and H. Treharne. Augmenting B with control annotations. In J. Julliand and O. Kouchnarenko, editors, B2007:Formal Specification and Development in B, volume 4355 of LNCS. Springer, January 2007.

[10] W. Ifill, I. Sorensen, and S. Schneider. High Integrity Software, chapter The Use of B to Specify, Design and Verify Hardware. Kluwer Academic Publishers, 2001.

[11] Alexandre Mota and Augusto Sampaio. Model-checking CSP-Z: Strategy, tool support and industrial application. Science of Computer Programming, 40(1):59-96, May 2001.

[12] J. D. Phillips and G. S. Stilles. An automatic translation of CSP to Handel-C. In I. East, J. Martin, P. Welch, D. Duce, and M. Green, editors, Communicating Process Architecures 2004. IOS Press, 2004, 2004.

[13] A. W. Roscoe. The Theory and Practice of Concurrency. Prentice-Hall, 1998.

[14] S. Schneider. Concurrent and Real-time Systems: The CSP Approach. John Wiley and Sons, 1999.

[15] S. Schneider. The B-Method: An introduction. Palgrave, 2002.

[16] S. Stepney. CSP/FDR2 to Handel-C translation. Technical report, University of York, June 2003.

[17] H. Treharne. Combining Control Executives and Software Specifications. PhD thesis, Royal Holloway, University of London, 2000.

[18] H. Treharne and S. Schneider. Communication B machines. In ZB2002, 2002. 


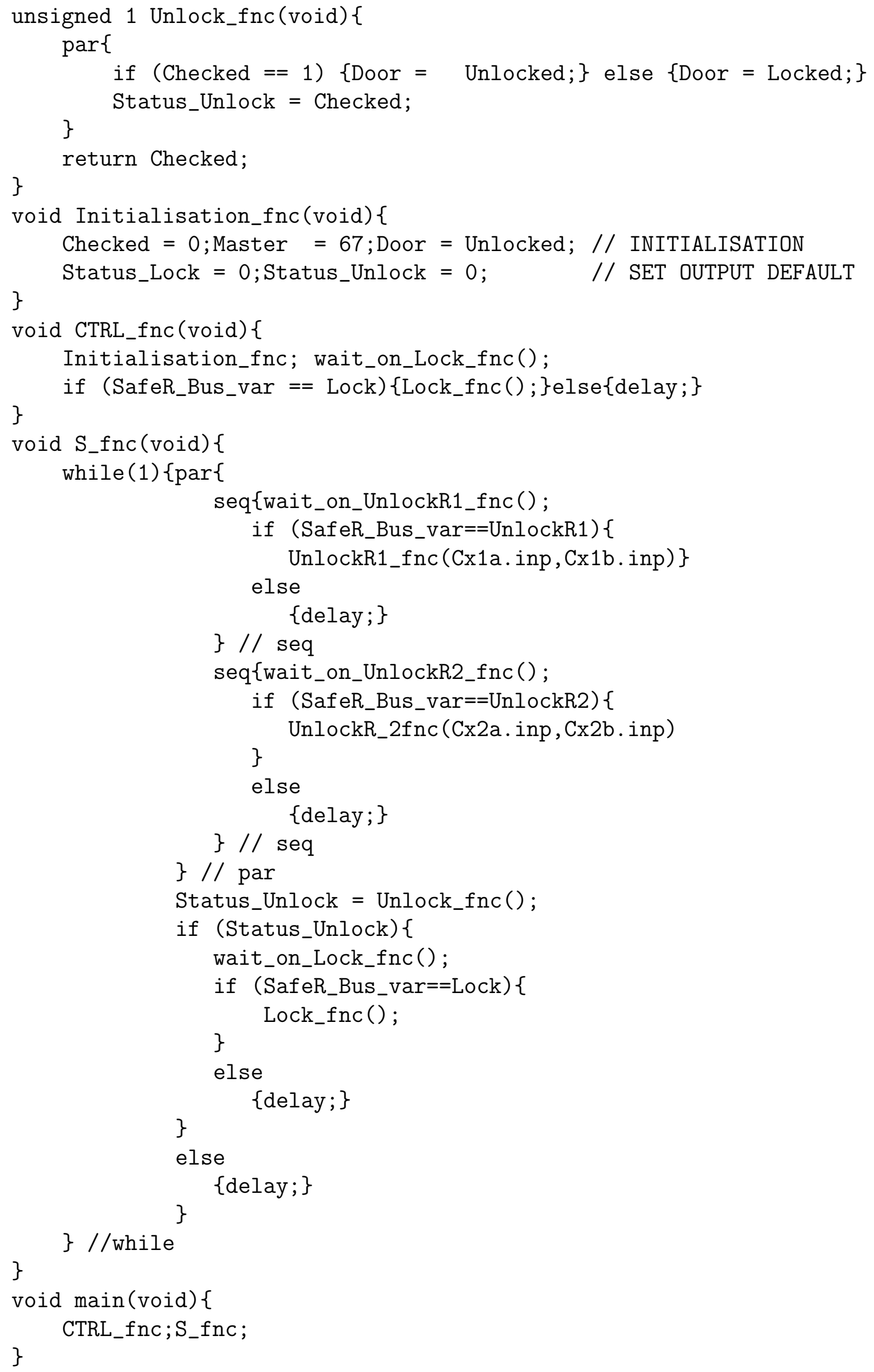

Figure 14. SafeR Translation Part 2. 


\begin{tabular}{|c|c|c|}
\hline Feature & $\mathrm{B}$ & Handel-C \\
\hline $\begin{array}{l}\text { set } \\
\text { declaration }\end{array}$ & $\begin{array}{l}\text { SETS SS }= \\
\mathrm{AA}, \ldots, \mathrm{XX} / *_{\mathrm{n}} * /\end{array}$ & $\begin{array}{l}\text { typedef enum }\{\mathrm{AA}= \\
\text { (unsigned } \mathrm{n} \text { ) } 0, \ldots, \mathrm{XX}\} \mathrm{SS} ;\end{array}$ \\
\hline \multirow[t]{3}{*}{$\begin{array}{l}\text { B variable } \\
\text { declaration }\end{array}$} & $\begin{array}{l}\text { INVARIANT } \\
\text { Vv } \in \text { TT /*OUTn*/ }\end{array}$ & $\begin{array}{l}\text { unsigned n Vv; } \\
\text { interface bus_out() } \\
\text { Vv1 (unsigned } 2 \text { OutPort=Vv); }\end{array}$ \\
\hline & $\begin{array}{l}\text { INVARIANT } \\
\text { Vv } \in \text { TT } / * I N n * /\end{array}$ & $\begin{array}{l}\text { unsigned n Vv; } \\
\text { interface bus_in(unsigned n inp) Vv(); }\end{array}$ \\
\hline & $\begin{array}{l}\text { INVARIANT } \\
\mathrm{Vv} \in \mathrm{TT} / *^{n} * /\end{array}$ & unsigned n Vv; \\
\hline $\begin{array}{l}\text { Function } \\
\text { Declaration }\end{array}$ & $\begin{array}{l}l^{*} \operatorname{extN}^{*} / \text { Oo } \\
\longleftarrow / * \operatorname{ext}^{*} / \mathbf{C c}(/ * \mathrm{M} * / \mathrm{Zz})\end{array}$ & 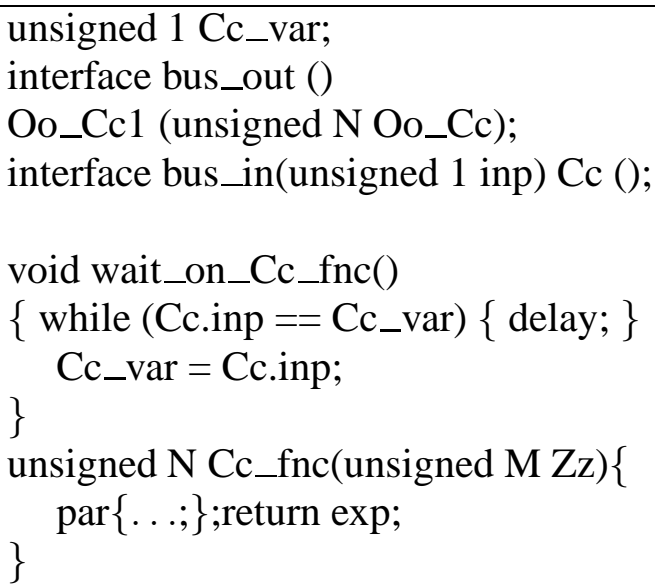 \\
\hline \multirow{3}{*}{$\begin{array}{l}\text { Function } \\
\text { Body }\end{array}$} & PRE $P$ THEN $B$ END & $\operatorname{par}\{<<B>>\}$ \\
\hline & IF $b$ THEN $c$ ELSE $d$ END & $\begin{array}{l}\text { if }<<b>>\{<<c>>\} \\
\text { else }\{<<d>>\}\end{array}$ \\
\hline & $b:=c$ & $<<b>>=<<c>>$ \\
\hline initialisation & INITIALISATION . . . & void Initialisation(void) $\{\ldots ;\}$ \\
\hline main & OPERATION & void main(void) $\{$ Initialisation; $\ldots\}$ \\
\hline
\end{tabular}

Figure 15. B to Handel-C Translation Guide.

\begin{tabular}{|c|c|c|}
\hline Feature & CSP & Handel-C \\
\hline $\begin{array}{l}\text { initialisation } \\
\text { processes }\end{array}$ & $\mathrm{P} \hat{=} \ldots \mathrm{R}$ & $\begin{array}{l}\text { P_fnc();Q_fnc(); } \\
\text { void P_fnc(void) }\{\ldots ;\}\end{array}$ \\
\hline $\begin{array}{l}\text { main loop } \\
\text { processes }\end{array}$ & $\mathrm{R}=\ldots \mathrm{R}$ & $\begin{array}{l}\text { R_fnc }() ; \\
\quad \text { void R_fnc(void) }\{\text { while }(1)\{\ldots ;\}\}\end{array}$ \\
\hline prefix (internal) & $<\mathrm{e} \rightarrow \mathrm{P}>$ & e_fnc $;<\mathrm{P}>$ \\
\hline prefix (external) & $<\mathrm{e} \rightarrow \mathrm{P}>$ & wait_on_e; e_fnc ; $<\mathrm{P}>$ \\
\hline choice (external) & $<\mathrm{P} 1 \square \mathrm{P} 2>$ & $<\mathrm{P} 1>$ \\
\hline interleaved & $\begin{array}{l}<e 1 \rightarrow \text { skip } \\
\|\| \ldots \| \\
e_{n} \rightarrow \text { skip } ; P>\end{array}$ & $\begin{array}{l}\mathrm{PAR}\left\{<e_{1} \rightarrow \text { skip }>\right. \\
\left.\ldots ;<e_{n} \rightarrow \text { skip }>\right\} ;<P>\end{array}$ \\
\hline \multirow[t]{2}{*}{ if-then-else } & $<$ if $\mathrm{y}$ then $\mathrm{P}$ else $\mathrm{Q}>$ & if $\mathrm{y}\{<\mathrm{P}\rangle\}$ else $\{<\mathrm{Q}>\}$ \\
\hline & $\begin{array}{l}\text { where }\langle P> \\
\text { is the translation of } \mathrm{P}\end{array}$ & \\
\hline
\end{tabular}

Figure 16. CSP to Handel-C Translation Guide. 